\title{
An Alternating Direction Implicit Finite Difference Method For Second Order Hyperbolic Equations
}

\author{
Jin Zhang ${ }^{1,2}$ \\ ${ }^{1}$ Business School, University of Shanghai for Science \\ and Technology, \\ Shanghai, China \\ ${ }^{2}$ School of Information Science and Engineering, \\ University of Jinan, \\ Jinan , China \\ e-mail: zdypaper@163.com
}

\author{
Weifeng Wang \\ Academic Affairs Office, University of Jinan, \\ Jinan , China
}

\begin{abstract}
In recent years, much attention has been given in the literature to the development, analysis, and implementation of finite difference schemes for the numerical solution of hyperbolic equations, which can describe physical phenomenons of vibrating string, elastic film and three-dimensional elastomers. In most of the cases, we use explicit difference schemes or implicit difference schemes to find numerical solutions of hyperbolic equations. The formers are suitable for parallel computation but it has limitations of stability. The latters are generally stable, but it is necessary to solve different linear systems at each level of time, which leads to more computational cost and time. In this paper, a two-level Crank-Nicolson alternating direction implicit (ADI) difference scheme is derived for solving the second order hyperbolic equations with variable coefficients by introducing the auxiliary variable. Convergence and stability analysis of the ADI scheme are given by the energy method. Finally, numerical examples are presented to illustrate the efficiency of the ADI difference scheme.
\end{abstract}

Keywords-implicit difference; alternating-direction; variable substitution; convergence; stability

\section{INTRODUCTION}

A large number of physical problems are modeled by the hyperbolic equations. For example, hyperbolic wave equations can describe physical phenomenons of vibrating string, elastic film and three-dimensional elastomers. In addition, the exploration of earth quake can be explained by some models related to non-linear hyperbolic equations. In recent years, much attention has been given to the development, analysis, and implementation of finite difference schemes for the numerical solution of secondorder hyperbolic equations. In [1]-[4], Mohanty has proposed some three level implicit unconditionally stable difference schemes for the one-, two- and threedimensional linear hyperbolic equations with constant and variable coefficients. Using nonpolynomial cubic spline in space and finite difference in time directions, Rashidinidia[5] constructed implicit three level difference schemes. In 2006, Gao[6] developed unconditionally stable difference schemes for the special one-dimensional second-order hyperbolic equation.

As is well known, the explicit difference schemes are suitable for parallel computation but it has limitations of stability. The implicit difference schemes are generally stable, but it is necessary to solve different linear systems at each level of time, which leads to more computational cost and time. In this paper, we propose a two-level alternating direction implicit difference scheme[7] for the following initial and boundary value problems of twodimensional hyperbolic equation:

$\left\{\begin{array}{l}u_{t t}-\nabla \cdot(a(x, y, t) \nabla u)=f(x, y, t) \\ (x, y, t) \in \Omega \times(0, T] \\ u(x, y, 0)=\varphi(x, y)(x, y) \in \Omega \\ u_{t}(x, y, 0)=\phi(x, y)(x, y) \in \Omega \\ u(x, y, t)=0(x, y) \in \Omega\end{array}\right.$

where $\Omega$ is a bounded domain in $R^{2}$ plane and $\Omega$ represents its boundary. $T$ is constant. $\nabla=\left\{\frac{\partial}{\partial x}, \frac{\partial}{\partial y}\right\}$. We suppose that there exist two constants $a_{*}>0$ and $a^{*}>0$ such that

$a_{*} \leq a(x, y, t) \leq a^{*}$

and

$a \in C^{3}(\overline{\Omega \times(0, T]}), u \in C^{6}(\overline{\Omega \times(0, T]})$

The alternating direction implicit (ADI) methods were first introduced by Douglas, Peaceman, and Rachford, which have an advantage of no increase in dimension of the coefficient matrices corresponding matrix equations. There are many extensions and a great variety of applications of ADI based on the finite difference or the finite element methods[8]-[12]. The energy method[13], which was first used for related theoretical analysis of alternating-direction scheme in [14], will be applied to the proofs of convergence and stability in this paper. 
Somenumerical examples will show that the proposed alternating-direction implicit scheme is stable and with an accuracy of second order.

\section{FORMULATION OF THE DIFFERENCE SCHEME}

Assume $\quad \Omega=(0,1) \times(0,1) \quad$ and $\quad$ let $\Delta x=\Delta y=h=1 / J, \Delta t=\tau=T / N$, where $J, N$ are positive integers. $\Delta x, \Delta y$ denote the grid spacing and $\Delta t$ denotes the time step. $x_{i}=i h, y_{j}=j h, i, j=0,1,2, \cdots, J$. $t^{n}=n \tau, n=0,1,2, \cdots, N$. For convenience, we shall adopt the following notations

$u^{n}=u_{i j}^{n}=u\left(x_{i}, y_{j}, t^{n}\right), \partial_{t}^{2} u^{n}=\frac{u^{n+1}-2 u^{n}+u^{n-1}}{\tau^{2}}$,

$\partial_{t} u^{n}=\frac{u^{n+1}-u^{n}}{\tau}, \partial_{\bar{t}} u^{n}=\frac{u^{n}-u^{n-1}}{\tau}, \partial_{\hat{t}} u^{n}=\frac{u^{n+1}-u^{n-1}}{2 \tau}$,

$\delta_{x}^{2} u_{i j}=\delta_{\bar{x}} \delta_{x} u_{i j}=\frac{u_{i+1, j}-2 u_{i j}+u_{i-1, j}}{h^{2}}$

$\delta_{x} u_{i j}=\frac{u_{i+1, j}-u_{i j}}{h}, \delta_{\bar{x}} u_{i j}=\frac{u_{i j}-u_{i-1, j}}{h}, \delta_{\hat{x}} u_{i j}=\frac{u_{i+1, j}-u_{i-1, j}}{2 h}$

$\bar{a}^{(1)}\left(w_{i j}^{n}\right)=\frac{1}{2}\left[a\left(w_{i+1, j}^{n}\right)+a\left(w_{i j}^{n}\right)\right]$,

$\delta_{\bar{x}}\left(a^{(1)}(w) \delta_{x} u\right)_{i j}^{n}=\frac{1}{h^{2}}\left[\bar{a}^{(1)}\left(w_{i j}^{n}\right)\left(u_{i+1, j}^{n}-u_{i j}^{n}\right)-\bar{a}^{(1)}\left(w_{i-1, j}^{n}\right)\left(u_{i j}^{n}-u_{i-1, j}^{n}\right)\right]$

$\nabla_{h} \cdot\left(a(w) \nabla_{h} u\right)_{i j}^{n}=\delta_{\bar{x}}\left(a^{(1)}(w) \delta_{x} u\right)_{i j}^{n}+\delta_{\bar{y}}\left(a^{(1)}(w) \delta_{y} u\right)_{i j}^{n}$

$\nabla_{h}=\left\{\delta_{x}, \delta_{y}\right\}, \Delta_{h}=\delta_{x}^{2}+\delta_{y}^{2}, \bar{\nabla}_{h}=\left\{\delta_{\hat{x}}, \delta_{\hat{y}}\right\}$

$\delta_{y} u_{i j}, \delta_{\bar{y}} u_{i j}, \delta_{\hat{y}} u_{i j}, \delta_{y}^{2} u_{i j}, \delta_{\bar{y}}\left(a^{(2)}(w) \delta_{y} u\right)_{i j}^{n}$ can be defined

in a similar way. Let $u_{t}=v$ and the first equation of (1) can be rewritten as

$\left\{\begin{array}{l}v_{t}-\nabla \cdot(a \nabla u)=f \\ u_{t}=v\end{array}\right.$

We obtain the Crank-Nicolson scheme(the subscripts are omitted)

$\left\{\begin{array}{l}\partial_{t} V^{n}-\nabla_{h} \cdot\left(a^{n+\frac{1}{2}} \nabla_{h} \frac{U^{n+1}+U^{n}}{2}\right)=f^{n+\frac{1}{2}} \\ \partial_{t} U^{n}=\frac{1}{2}\left(V^{n+1}+V^{n}\right)\end{array}\right.$

the following equation can be obtained by eliminating $U^{n+1}$

$\partial_{t} V^{n}-\frac{\tau}{2} \nabla_{h} \cdot\left(a^{n+\frac{1}{2}} \nabla_{h} \frac{V^{n+1}+V^{n}}{2}\right)=f^{n+\frac{1}{2}}+\nabla_{h} \cdot\left(a^{n+\frac{1}{2}} \nabla_{h} U^{n}\right)$

Finally, we obtained the alternating-direction difference scheme based on Peaceman-Rachford scheme:

$$
\left\{\begin{array}{l}
\frac{V^{n+\frac{1}{2}}-V^{n}}{\tau / 2}-\frac{\tau}{2} \delta_{\bar{x}}\left(\bar{a}^{(1) n+\frac{1}{2}} \delta_{x} V^{n+\frac{1}{2}}\right)-\frac{\tau}{2} \delta_{\bar{y}}\left(\bar{a}^{(2) n+\frac{1}{2}} \delta_{y} V^{n}\right)=f^{n+\frac{1}{2}}+\nabla_{h} \cdot\left(a^{n+\frac{1}{2}} \nabla_{h} U^{n}\right) \\
\frac{V^{n+1}-V^{n+\frac{1}{2}}}{\tau / 2}-\frac{\tau}{2} \delta_{\bar{x}}\left(\bar{a}^{(1) n+\frac{1}{2}} \delta_{x} V^{n+\frac{1}{2}}\right)-\frac{\tau}{2} \delta_{\bar{y}}\left(\bar{a}^{(2) n+\frac{1}{2}} \delta_{y} V^{n+1}\right)=f^{n+\frac{1}{2}}+\nabla_{h} \cdot\left(a^{n+\frac{1}{2}} \nabla_{h} U^{n}\right) \\
\partial_{t} U^{n}=\frac{1}{2}\left(V^{n+1}+V^{n}\right)
\end{array}\right.
$$

\section{THE ANALYSIS OF CONVERGENCE AND STABILITY}

For convenience, we define the following inner products and related norms:

$$
\begin{aligned}
& (\mathrm{u}, \mathrm{v})=\sum_{i=1}^{J-1} \sum_{j=1}^{J-1} u_{i j} v_{i j} h^{2},\|u\|^{2}=(u, u) \\
& {\left[\nabla_{h} u, \nabla_{h} v\right)=\sum_{i=0}^{J-1} \sum_{j=1}^{J-1} \delta_{x} u_{i j} \delta_{y} v_{i j} h^{2}+\sum_{i=1}^{J-1} \sum_{j=0}^{J-1} \delta_{x} u_{i j} \delta_{y} v_{i j} h^{2},} \\
& |u|_{1}^{2}=\left[\nabla_{h} u, \nabla_{h} v\right) \\
& (u, v)_{1 x}=\sum_{i=0}^{J-1} \sum_{j=0}^{J-1} \delta_{x} u_{i j} \delta_{x} v_{i j} h^{2}, \\
& |u|_{1 x}^{2}=(u, v)_{1 x} \\
& (u, v)_{1 y}=\sum_{i=0}^{J-1} \sum_{j=0}^{J-1} \delta_{y} u_{i j} \delta_{y} v_{i j} h^{2}, \\
& |u|_{1 y}^{2}=(u, v)_{1 y} \\
& \left\|\nabla_{h} u\right\|^{2}=|u|_{1 x}^{2}+|u|_{1 y}^{2}, \\
& \|u\|_{\infty}=\max _{0 \leq i, j \leq J} u_{i j} \mid \\
& (\mathrm{u}, \mathrm{v})_{2}=\sum_{i=0}^{J-1} \sum_{j=0}^{J-1} \delta_{x} \delta_{y} u_{i j} \delta_{x} \delta_{y} v_{i j} h^{2},|u|_{2}^{2}=(u, u)_{2}
\end{aligned}
$$

It is easy to show that the following two lemmas hold:

\section{Lemma 1.}

$$
\begin{aligned}
& \forall\left(x_{i}, y_{j}\right) \in \Omega, u_{i j}=v_{i j}=0 \Rightarrow \\
& \left\{\begin{array}{l}
\sum_{i=1}^{J-1} \sum_{j=1}^{J-1} \delta_{\bar{x}}\left(a^{(1)} \delta_{x} u_{i j}\right) \cdot v_{i j} \cdot h^{2}=-\sum_{i=0}^{J-1} \sum_{j=1}^{J-1} a_{i+\frac{1}{2}, j} \cdot \delta_{x} u_{i j} \cdot \delta_{x} v_{i j} \cdot h^{2} \\
\sum_{i=1}^{J-1} \sum_{j=1}^{J-1} \delta_{\bar{y}}\left(a^{(2)} \delta_{y} u_{i j}\right) \cdot v_{i j} \cdot h^{2}=-\sum_{i=1}^{J-1} \sum_{j=0}^{J-1} a_{i, j+\frac{1}{2}} \cdot \delta_{y} u_{i j} \cdot \delta_{y} v_{i j} \cdot h^{2}
\end{array}\right.
\end{aligned}
$$

\section{Lemma 2.}

$$
\left\{\begin{array}{l}
\delta_{x}\left(u_{i j} \cdot v_{i j}\right)=u_{i j} \cdot \delta_{x} v_{i j}+\delta_{x} u_{i j} \cdot v_{i+1, j} \\
\delta_{x} \delta_{y}\left(u_{i j} \cdot v_{i j}\right)=u_{i j} \cdot \delta_{x} \delta_{y} v_{i j}+\delta_{x} u_{i j} \cdot \delta_{y} v_{i+1, j}+\delta_{y} u_{i j} \cdot \delta_{x} v_{i, j+1}+\delta_{x} \delta_{y} u_{i j} \cdot v_{i+1, j+1}
\end{array}\right.
$$


Theorem 1. (Convergence) Let $u$ be the exact solution of (1) and $U$ be the approximate solution of (7). If (2) and (3) are satisfied, then there exists a constant $K$ such that $\left\|v^{k}-V^{k}\right\|^{2}+\left\|\nabla_{h}\left(u^{k}-U^{k}\right)\right\|^{2} \leq K\left(\tau^{4}+h^{4}\right)$ $k \in Z^{+}, K \in R^{+}$

Proof. We can obtain the following system of equations from (7) by eliminating $V^{n+\frac{1}{2}}$

$$
\left\{\begin{array}{l}
\partial_{t} V^{n}-\nabla_{h} \cdot\left(a^{n+\frac{1}{2}}\right) \nabla_{h} \frac{U^{n+1}+U^{n}}{2}+ \\
\frac{\tau^{3}}{16} \delta_{\bar{x}}\left(\bar{a}^{(1)} \delta_{x}\right) \delta_{\bar{y}}\left(\bar{a}^{(2)} \delta_{y}\right)\left(V^{n+1}-V^{n}\right)=f^{n+\frac{1}{2}} \\
\partial_{t} U^{n}=\frac{1}{2}\left(V^{n+1}+V^{n}\right)
\end{array}\right.
$$

From (4) we have

$$
\left\{\begin{array}{l}
\partial_{t} v^{n}-\nabla_{h} \cdot\left(a^{n+\frac{1}{2}}\right) \nabla_{h} \frac{u^{n+1}+u^{n}}{2}+ \\
\frac{\tau^{3}}{16} \delta_{\bar{x}}\left(\bar{a}^{(1)} \delta_{x}\right) \delta_{\bar{y}}\left(\bar{a}^{(2)} \delta_{y}\right)\left(v^{n+1}-v^{n}\right)=f^{n+\frac{1}{2}}+R_{1} \\
\partial_{t} u^{n}=\frac{1}{2}\left(v^{n+1}+v^{n}\right)+R_{2}
\end{array}\right.
$$

where

$$
\begin{gathered}
R_{1}=\partial_{t} v^{n}-v_{t}^{n+\frac{1}{2}}+\nabla \cdot\left(a^{n+\frac{1}{2}} \nabla u^{n+\frac{1}{2}}\right)-\nabla_{h} \cdot\left(a^{n+\frac{1}{2}} \nabla_{h} \frac{u^{n+1}+u^{n}}{2}\right) \\
+\frac{\tau^{3}}{16} \delta_{\bar{x}}\left(\bar{a}^{(1)} \delta_{x}\right) \delta_{\bar{y}}\left(\bar{a}^{(2)} \delta_{y}\right)\left(v^{n+1}-v^{n}\right) \\
R_{2}=\partial_{t} u^{n}-u_{t}^{n+\frac{1}{2}}+v^{n+\frac{1}{2}}-\frac{1}{2}\left(v^{n+1}+v^{n}\right)
\end{gathered}
$$

Let $e^{n}=u^{n}-U^{n}$ and $\theta^{n}=v^{n}-V^{n}$. From (11) and (12), we can obtain the error equations

$$
\left\{\begin{array}{l}
\partial_{t} \theta^{n}-\nabla_{h} \cdot\left(a^{n+\frac{1}{2}} \nabla_{h} \frac{e^{n+1}+e^{n}}{2}\right)+ \\
\frac{\tau^{3}}{16} \delta_{\bar{x}}\left(\bar{a}^{(1)} \delta_{x}\right) \delta_{\bar{y}}\left(\bar{a}^{(2)} \delta_{y}\right)\left(\theta^{n+1}-\theta^{n}\right)=R_{1} \\
\partial_{t} e^{n}=\frac{1}{2}\left(\theta^{n+1}+\theta^{n}\right)+R_{2}
\end{array}\right.
$$

Taking the inner product (.,.) on both sides of the first error equation with $\left(\theta^{n+1}+\theta^{n}\right) \tau$, we have

$$
\begin{aligned}
& \left(\theta^{n+1}-\theta^{n}, \theta^{n+1}+\theta^{n}\right)-\left(\nabla_{h} \cdot\left(a^{n+\frac{1}{2}} \nabla_{h}\left(e^{n+1}+e^{n}\right)\right), e^{n+1}-e^{n}\right) \\
& \quad+\frac{\tau^{4}}{16}\left(\delta_{\bar{x}}\left(\bar{a}^{(1)} \delta_{x}\right) \delta_{\bar{y}}\left(\bar{a}^{(2)} \delta_{y}\right)\left(\theta^{n+1}-\theta^{n}\right), \theta^{n+1}+\theta^{n}\right) \\
& =\left(\nabla_{h} \cdot\left(a^{n+\frac{1}{2}} \nabla_{h}\left(e^{n+1}+e^{n}\right)\right), R_{2}\right) \tau+\left(R_{1}, \theta^{n+1}+\theta^{n}\right) \tau
\end{aligned}
$$

Applying the operator $\nabla_{h}$ to both sides of the second equation of (13) and taking the inner product (.,.) on both sides of (13) with $\nabla_{h}\left(\theta^{n+1}+\theta^{n}\right)$, gives

$\left(\nabla_{h} \partial_{t} e^{n}, \nabla_{h}\left(\theta^{n+1}+\theta^{n}\right)\right)=\frac{1}{2}\left(\nabla_{h}\left(\theta^{n+1}+\theta^{n}\right), \nabla_{h}\left(\theta^{n+1}+\theta^{n}\right)\right)$

$+\left(\nabla_{h} R_{2}, \nabla_{h}\left(\theta^{n+1}+\theta^{n}\right)\right)$

Notice that $\left(\theta^{n+1}+\theta^{n}\right)=2 \partial_{t} e^{n}-2 R_{2}$, we obtain from (14)

$\left\|\nabla_{h}\left(\theta^{n+1}+\theta^{n}\right)\right\|^{2}=4\left\|\nabla_{h} \partial_{t} e^{n}\right\|^{2}-8\left(\nabla_{h} \partial_{t} e^{n}, \nabla_{h} R_{2}\right)$

$+4\left\|\nabla_{h} R_{2}\right\|^{2}$

We will estimate the left-hand side terms of (14) term by term. The first item is

$\left(\theta^{n+1}-\theta^{n}, \theta^{n+1}+\theta^{n}\right)=\left\|\theta^{n+1}\right\|^{2}-\left\|\theta^{n}\right\|^{2}$

The second inner product can be rewritten as the sum of two terms and the part in $x$-direction is as follows(note that the boundary value of $e^{n}$ is zero), so does29n ydirection.

$$
\begin{gathered}
-\left(\delta_{\bar{x}}\left(\bar{a}^{(1)} \delta_{x}\right)\left(e^{n+1}+e^{n}\right), e^{n+1}-e^{n}\right) \\
\sum_{i=0}^{J-1} \sum_{j=0}^{J-1} a^{n+\frac{1}{2}}\left(\delta_{x} e_{i j}^{n+1}\right)^{2} h^{2}-\sum_{i=0}^{J-1} \sum_{j=0}^{J-1} a^{n-\frac{1}{2}}\left(\delta_{x} e_{i j}^{n}\right)^{2} h^{2} \\
-\sum_{i=0}^{J-1} \sum_{j=0}^{J-1} \frac{a^{n+\frac{1}{2}}-a^{n-\frac{1}{2}}}{\tau}\left(\delta_{x} e_{i j}^{n}\right)^{2} h^{2}
\end{gathered}
$$

The third inner product is decomposed into four items by using Lemma 1 and Lemma 2,

$$
\begin{gathered}
\quad \frac{\tau^{4}}{16}\left(\delta_{\bar{x}}\left(\bar{a}^{(1)} \delta_{x}\right) \delta_{\bar{y}}\left(\bar{a}^{(2)} \delta_{y}\right)\left(\theta^{n+1}-\theta^{n}\right), \theta^{n+1}+\theta^{n}\right) \\
=\frac{\tau^{4}}{16} \sum_{i, j=0}^{J-1} a_{i, j+\frac{1}{2}} \cdot \delta_{x} \delta_{y}\left(\theta_{i j}^{n+1}-\theta_{i j}^{n}\right) \cdot a_{i+\frac{1}{2}, j} \cdot \delta_{y} \delta_{x}\left(\theta_{i j}^{n+1}+\theta_{i j}^{n}\right) \cdot h^{2} \\
+\frac{\tau^{4}}{16} \sum_{i, j=0}^{J-1} a_{i, j+\frac{1}{2}} \cdot \delta_{x} \delta_{y}\left(\theta_{i j}^{n+1}-\theta_{i j}^{n}\right) \cdot \delta_{y} a_{i+\frac{1}{2}, j} \cdot \delta_{x}\left(\theta_{i, j+1}^{n+1}+\theta_{i, j+1}^{n}\right) \cdot h^{2} \\
+\frac{\tau^{4}}{16} \sum_{i, j=0}^{J-1} \delta_{x} a_{i, j+\frac{1}{2}} \cdot \delta_{y}\left(\theta_{i+1, j}^{n+1}-\theta_{i+1, j}^{n}\right) \cdot a_{i+\frac{1}{2}, j} \cdot \delta_{y} \delta_{x}\left(\theta_{i j}^{n+1}+\theta_{i j}^{n}\right) \cdot h^{2} \\
+\frac{\tau^{4}}{16} \sum_{i, j=0}^{J-1} \delta_{x} a_{i, j+\frac{1}{2}} \cdot \delta_{y}\left(\theta_{i+1, j}^{n+1}-\theta_{i+1, j}^{n}\right) \cdot \delta_{y} a_{i+\frac{1}{2}, j} \cdot \delta_{x}\left(\theta_{i, j+1}^{n+1}+\theta_{i, j+1}^{n}\right) \cdot h^{2}
\end{gathered}
$$


For convenience, the four items of the right-hand side of the above equation will be denoted by B1, B2, B3, and B4, respectively. Similar to (18),

$$
\begin{aligned}
& B_{1}=\frac{\tau^{4}}{16}\left\{\sum_{i=0}^{J-1} \sum_{j=0}^{J-1} A^{n+\frac{1}{2}}\left(\delta_{x} \delta_{y} \theta_{i j}^{n+1}\right)^{2} h^{2}-\right. \\
& \sum_{i=0}^{J-1} \sum_{j=0}^{J-1} A^{n-\frac{1}{2}}\left(\delta_{x} \delta_{y} \theta_{i j}^{n}\right)^{2} h^{2}-
\end{aligned}
$$

$\left.\sum_{i=0}^{J-1} \operatorname{sum}_{j=0}^{J-1} \frac{A^{n+\frac{1}{2}}-A^{n-\frac{1}{2}}}{\tau}\left(\delta_{x} \delta_{y} \theta_{i j}^{n}\right)^{2} h^{2}\right\}$

where $A^{n+\frac{1}{2}}=a_{i+\frac{1}{2}, j}^{n+\frac{1}{2}} \cdot a_{i, j+\frac{1}{2}}^{n+\frac{1}{2}}$. Assume $\mathrm{K}$ be a generic constant, which maybe different at different places. Based on the boundedness of $a$ and (16), we have

$$
\begin{array}{cc}
-B_{2} & \leq K \tau^{4}\left(\left|\theta^{n+1}\right|_{2}^{2}+\left|\theta^{n}\right|_{2}^{2}\right) \tau+K\left(\left\|\nabla_{h} e^{n+1}\right\|^{2}+\left\|\nabla_{h} e^{n}\right\|^{2}\right) \tau+K \tau^{5} \\
-B_{3} & \leq K \tau^{4}\left(\left|\theta^{n+1}\right|_{2}^{2}+\left|\theta^{n}\right|_{2}^{2}\right) \tau+K\left(\left\|\nabla_{h} e^{n+1}\right\|^{2}+\left\|\nabla_{h} e^{n}\right\|^{2}\right) \tau+K \tau^{5} \\
-B_{4} & \leq K\left(\left\|\nabla_{h} e^{n+1}\right\|^{2}+\left\|\nabla_{h} e^{n}\right\|^{2}\right) \tau+K \tau^{5}
\end{array}
$$

The estimates of right-hand side terms of (14) are similar to that of left-hand side and we have

$$
\begin{aligned}
& \left(\nabla_{h} \cdot\left(a^{n+\frac{1}{2}} \nabla_{h}\left(e^{n+1}+e^{n}\right)\right), R_{2}\right) \tau \leq K\left(\left\|\nabla_{h} e^{n+1}\right\|^{2}+\left\|\nabla_{h} e^{n}\right\|^{2}\right) \tau+K \tau \\
& \left(R_{1}, \theta^{n+1}+\theta^{n}\right) \tau \leq K \tau^{4}\left(\left|\theta^{n+1}\right|_{2}^{2}+\left|\theta^{n}\right|_{2}^{2}\right) \tau+K\left(\left\|\nabla_{h} e^{n+1}\right\|^{2}+\left\|\nabla_{h} e^{n}\right\|^{2}\right) \tau+ \\
& \left(\left\|\theta^{n+1}\right\|^{2}+\left\|\theta^{n}\right\|^{2}\right) \tau+K \tau^{5}+K h^{4} \tau
\end{aligned}
$$

Substituting the estimates (17)-(22) into (14) and summing for $\mathrm{n}=0$ to $\mathrm{n}=\mathrm{k}-1$, we obtain the following inequation

$$
\begin{gathered}
\left\|\theta^{k}\right\|^{2}+\tau^{4}\left|\theta^{k}\right|_{2}^{2}+\left\|\nabla_{h} e^{k}\right\|^{2} \\
\leq K\left(\sum_{n=0}^{k-1}\left\|\theta^{n}\right\|^{2} \tau+\tau^{4} \sum_{n=0}^{k-1}\left|\theta^{n}\right|_{2}^{2} \tau+\sum_{n=0}^{k-1}\left\|\nabla_{h} e^{n}\right\|^{2} \tau+\left(\tau^{4}+h^{4}\right)\right)
\end{gathered}
$$

By using the Gronwall's Lemma, the error estimate is followed:

$\left\|v^{k}-V^{k}\right\|^{2}+\left\|\nabla_{h}\left(u^{k}-U^{k}\right)\right\|^{2} \leq K\left(\tau^{4}+h^{4}\right)$

By using similar arguments as those in the proofs of Theorem 1, we obtain the following stability estimate Theorem 2. Stability

$\left\|V^{k}\right\|^{2}+\left\|\nabla_{h} U^{k}\right\|^{2} \leq K\left(\left\|V^{0}\right\|^{2}+\left|V^{0}\right|_{2}^{2}+\left\|\nabla_{h} U^{0}\right\|^{2}+\sum_{n=0}^{k}\left\|f^{n+\frac{1}{2}}\right\|^{2} \tau\right)$

\section{NUMERICAL RESULTS}

We will use our scheme for solving some hyperbolic equations. $u(x, y, t)=1 / 4 \sin (x y(1-x)(1-y))(3 / 2+\cos (2 t))$ is the exact solution of (1), where $a=u+1 / 2$. Then $u_{t}$ is replaced by $v$ and $v(x, y, t)=-1 / 2 \sin (x y(1-x)(1-y)) \sin (2 t)$. MATLAB program based on the corresponding alternating-direction scheme (7) is performed and the difference solution is obtained. The comparison between the exact solution and the approximate solution show that our scheme is effective.

\begin{tabular}{|c|c|c|c|c|c|c|c|}
\hline $\mathrm{t}$ & \multirow{2}{*}{$\begin{array}{c}\gamma \\
\gamma_{0}\end{array}$} & \multirow{2}{*}{$\frac{\|e\| /\|u\|}{9.5811 \mathrm{e}-3}$} & \multirow[t]{2}{*}{ rate } & \multirow{2}{*}{$\frac{\|e\|_{\infty} /\|u\|_{\infty}}{1.0487 \mathrm{e}-2}$} & rate & $|e|_{1} /|u|_{1}$ & rate \\
\hline 5 & & & & & & $9.5355 \mathrm{e}-3$ & \\
\hline & $\gamma_{0} / 2$ & $2.2036 \mathrm{e}-3$ & 2.2472 & $2.2743 \mathrm{e}-3$ & 2.3323 & $2.1931 \mathrm{e}-3$ & 2.2388 \\
\hline 5 & $\gamma_{0} / 4$ & $5.4115 \mathrm{e}-4$ & 2.0877 & $5.5083 \mathrm{e}-4$ & 2.1077 & $5.3961 \mathrm{e}-4$ & 2.0828 \\
\hline \multirow[t]{3}{*}{10} & $\gamma_{0}$ & $5.5985 \mathrm{e}-3$ & & $6.2752 \mathrm{e}-3$ & & $5.5916 \mathrm{e}-3$ & \\
\hline & $\gamma_{0} / 2$ & $1.0139 \mathrm{e}-3$ & 2.3862 & $1.0546 \mathrm{e}-3$ & 2.4944 & $1.0090 \mathrm{e}-3$ & 2.3830 \\
\hline & $\gamma_{0} / 4$ & $2.2356 \mathrm{e}-4$ & 2.1442 & $2.5508 \mathrm{e}-4$ & 2.0107 & $2.2299 \mathrm{e}-4$ & 2.1387 \\
\hline \multirow[t]{3}{*}{15} & $\gamma_{0}$ & $1.2432 \mathrm{e}-2$ & & $1.2751 \mathrm{e}-2$ & & $1.2384 \mathrm{e}-2$ & \\
\hline & $\gamma_{0} / 2$ & $2.8721 \mathrm{e}-3$ & 2.1901 & $2.9591 \mathrm{e}-3$ & 2.1839 & $2.8584 \mathrm{e}-3$ & 2.1829 \\
\hline & $\gamma_{0} / 4$ & $7.0004 \mathrm{e}-4$ & 2.0772 & $7.4577 \mathrm{e}-4$ & 2.0290 & $6.9558 \mathrm{e}-4$ & 2.0774 \\
\hline
\end{tabular}
Table 1 displays the errors and shows that the convergence rate of the proposed ADI scheme is second order, where $\gamma=(\tau, h), \gamma_{0}=(0.1,1 / 8)$. The 3th, 5th and 7 th column are the relative errors to corresponding norms and the 4th, 6 th and 8th are convergence rates to corresponding norms. Three figures illustrate the numerical solution(left) and the exact solution(right) at different times at $t=10 . \tau=0.05, h=1 / 16$

TABLE I. ERRORS AND CONVERGENCE RATE

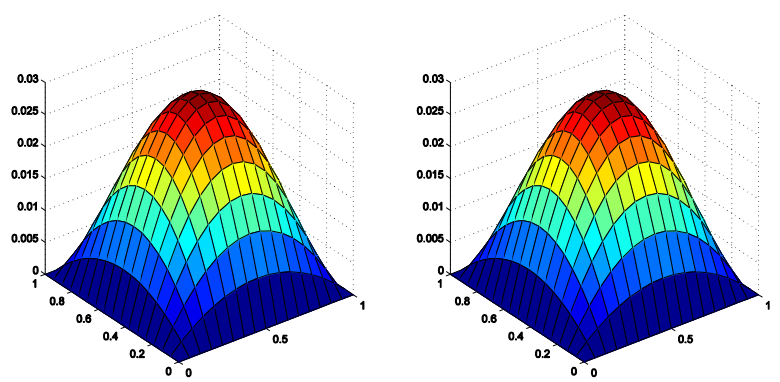

Figure 1. The profile of numerical solution(left) and the exact solution(right) when $\Omega=(0,1) \times(0,1)$

$k \in Z^{+}, K \in R^{+}$ 

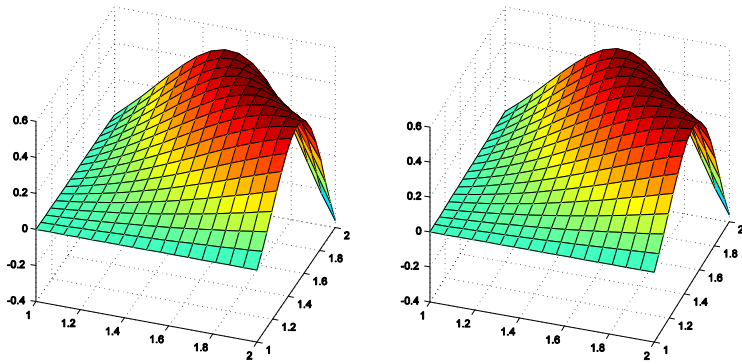

Figure 2. The profile of numerical solution(left) and the exact solution(right) when $\Omega=(1,2) \times(1,2)$

\section{CONCLUSION}

A two-level Crank-Nicolson alternating direction implicit difference scheme is derived for solving the second order hyperbolic equations with variable coefficients by introducing the auxiliary variable $v$. Convergence and stability analysis show that the ADI scheme is absolutely stable and have a convergence rate of order $O\left(\tau^{2}+h^{2}\right)$. Numerical experiment indicates that the proposed scheme is easy for parallel computing, stable, and effective.

\section{REFERENCES}

[1] R.K. Mohanty, An unconditionally stable finite difference formula for a linear second order one space dimensional hyperbolic equation with variable coefficients, Appl. Math. Comput. 165 (2005) 229-236.

[2] R.K. Mohanty, An unconditionally stable difference scheme for the one-space-dimensional linear hyperbolic equation, Appl. Math. Lett. 17 (2001)101-105.
[3] R.K. Mohanty, M.K. Jam, An unconditionally stable alternating direction implicit scheme for the two space dimensional linear hyperbolic equation, Numer. Methods Partial Differential Equations 17 (2001) 684-688.

[4] R.K. Mohanty, M.K. Jain, U. Arora, An unconditionally stable ADI method for the linear hyperbolic equation in three space dimensional, Int. Appl. J.Comput. Math. 79(2002): 133-142.

[5] J. Rashidinia, R. Mohammadi, R. Jalilian, Spline methods for the solution of hyperbolic equation with variable coefficients, Numer. Methods Partial Differential Equations 32(2006):1-9.

[6] Gao F, Chi C. Unconditionally stable difference schemes for a onespace-dimensional linear hyperbolic equation. Applied Mathematics and Computation, 2007, 187(2): 1272-1276.

[7] Peaceman D W, Rachford, Jr H H. The numerical solution of parabolic and elliptic differential equations[J]. Journal of the Society for Industrial \& Applied Mathematics, 1955, 3(1): 28-41.

[8] Douglas J. Alternating direction methods for three space variables. Numerische Mathematik, 1962, 4(1): 41-63.

[9] Douglas J, Gunn J E. A general formulation of alternating direction methods. Numerische Mathematik, 1964, 6(1): 428-453.

[10] Douglas Jr J, Dupont T. Alternating-direction Galerkin methods on rectangles. Numerical Solution of Partial Differential Equations, II (SYNSPADE 1970), 1971: 133-214.

[11] Namiki T. A new FDTD algorithm based on alternating-direction implicit method. Microwave Theory and Techniques, IEEE Transactions on, 1999, 47(10): 2003-2007.

[12] Staker S W, Holloway C L, Bhobe A U, et al. Alternating-direction implicit (ADI) formulation of the finite-difference time-domain (FDTD) method: algorithm and material dispersion implementation. Electromagnetic Compatibility, IEEE Transactions on, 2003, 45(2): 156-166.

[13] Lees M. A priori estimates for the solutions of difference approximations to parabolic partial differential equations. Duke Mathematical Journal, 1960, 27(3): 297-311.

[14] Lees M. Alternating direction and semi-explicit difference methods for parabolic partial differential equations. Numerische Mathematik, 1961, 3(1): 398-412. 